\title{
Autophagy-dependent cell death
}

\author{
Donna Denton $\mathbb{1}^{1} \cdot$ Sharad Kumar ${ }^{1}$
}

Received: 20 October 2018 / Revised: 9 November 2018 / Accepted: 28 November 2018 / Published online: 19 December 2018

(c) ADMC Associazione Differenziamento e Morte Cellulare 2018

\begin{abstract}
Autophagy-dependent cell death can be defined as cell demise that has a strict requirement of autophagy. Although autophagy often accompanies cell death following many toxic insults, the requirement of autophagic machinery for cell death execution, as established through specific genetic or chemical inhibition of the process, is highly contextual. During animal development, perhaps the best validated model of autophagy-dependent cell death is the degradation of the larval midgut during larval-pupal metamorphosis, where a number of key autophagy genes are required for the removal of the tissues. Surprisingly though, even in the midgut, not all of the 'canonical' autophagic machinery appears to be required. In other organisms and cancer cells many variations of autophagy-dependent cell death are apparent, pointing to the lack of a unifying cell death pathway. It is thus possible that components of the autophagy machinery are selectively utilised or repurposed for this type of cell death. In this review, we discuss examples of cell death that utilise autophagy machinery (or part thereof), the current knowledge of the complexity of autophagy-dependent cellular demise and the potential mechanisms and regulatory pathways involved in such cell death.
\end{abstract}

\section{Facts}

- Autophagy often accompanies cell death, but autophagy-dependent cell death is highly contextual.

- It utilises key components of the macroautophagy machinery.

- At least in Drosophila development, such cell death is highly regulated.

- Growth arrest often precedes autophagy-dependent cell death.

\section{Open questions}

- What are the key signals that initiate autophagydependent cell death?

Edited by F. Pentimallli

Donna Denton

donna.denton@unisa.edu.au

Sharad Kumar

sharad.kumar@unisa.edu.au

1 Centre for Cancer Biology, University of South Australia and SA Pathology, GPO Box 2471, Adelaide, SA 5001, Australia
- What are the mechanisms of cell death dependent on autophagy?

- Is there a unified mechanism of autophagy-dependent cell death?

- Is the machinery required for autophagy induced as a cell survival response the same as that mediating autophagy dependent cell death?

- How prevalent is autophagy-dependent cell death and what is its evolutionary significance?

\section{Introduction}

Cell death plays an essential role in maintaining organismal homoeostasis by deleting excess and unwanted cells. Most cell deaths utilise a caspase-dependent pathway, commonly known as apoptosis. Genetic studies in model systems suggest that apoptosis accounts for the bulk of developmentally programmed cell death (PCD) [1-4]. For example, in Caenorhabditis elegans loss of function mutations in key apoptosis genes, such as ced-3 and ced-4, block the death of all 131 somatic cells during development [5, 6]. In mice, however, blocking apoptosis appears to have only a limited effect on development [7-9], presumably due to compensatory backup mechanisms ensuring that unwanted cells get deleted by nonapoptotic means. 
In addition to apoptosis, a number of regulated cell death modalities have come to light in recent years [10]. Among these, those that occur during development and homoeostasis include apoptosis, necroptosis (also called programmed necrosis) and autophagy-dependent cell death [10]. Apoptosis is characterised by a highly orchestrated fragmentation of a cell into apoptotic bodies, which are recognised and removed rapidly by phagocytosis. The apoptosis execution occurs by two pathways, the intrinsic and extrinsic, that centre on the activation of cysteineproteases called caspases [11]. The intrinsic pathway is activated by a developmental program and by external signals, such as ionising radiation, growth factor depletion or glucocorticoids, releasing pro-apoptotic factors from the mitochondria. The extrinsic pathway is triggered by extracellular ligands binding to membrane-localised death receptors. Necroptosis is characterised by organelle swelling and lysis, inflammation and release of intracellular material in response to pathological conditions. Autophagy is a conserved process that delivers components of the cytoplasm to lysosomes for degradation. While originally identified as a cell survival mechanism, autophagy plays highly context-specific roles in mediating cell death $[12,13]$. The interplay between autophagy-dependent cell death and other types of cell deaths have been reviewed recently [14]. Here, we focus on various physiological examples of autophagy-dependent cell death, review the potential mechanisms and regulation of such death, as well as discuss its potential significance.

\section{Autophagy}

Autophagy is the process that delivers cytoplasmic material of endogenous or exogenous origin to the lysosome for degradation [15]. There are three main forms of autophagy, known as macroautophagy (herein referred to as autophagy), microautophagy and chaperone-mediated autophagy (CMA) [15]. Microautophagy involves the transfer of cytosolic cargo components directly into the lysosome through membrane invaginations $[15,16]$. CMA involves the selective translocation of proteins containing the KFERQ-like motif across the lysosomal membrane [17].

The evolutionarily conserved process of autophagy is carried out by a molecular machinery encoded by Autophagy-related (Atg) genes originally identified from genetic screens in yeast [18-21]. Detailed description of the molecular machinery regulating autophagy can be found in many reviews including refs. [15, 22-25]. Briefly, initiation of autophagy requires the Atg1/Unc-51-like kinase (ULK) complex [26, 27]. The function of the Atg1/ULK1 complex is to transduce the signals which induce its recruitment to the isolation membrane by phosphorylating and/or recruiting other proteins necessary for the autophagosome formation. This recruits the class III PI3K/PtdIns(3)K complex, containing vacuolar protein sorting 34 (VPS34/ PIK3C3), Vps15 (PIK3R4), Atg6 (BECLIN1) and Atg14, necessary for phosphatidylinositol 3-phosphate (PI(3)P) production and subsequent recruitment of autophagy factors to the autophagosomal membrane [28]. Two conserved ubiquitin-like conjugation systems (Atg8/LC3-lipid phosphatidylethanolamine (PE) and Atg12-Atg5 systems) mediate insertion of lipidated Atg8 (including LC3 and GABARAP family members in mammals) into the autophagosomal membrane during elongation and expansion to form a fully closed autophagosome [29-31]. The final step in autophagy is the fusion of the autophagosome with the lysosome to form an autolysosome for degradation/recycling of cellular components [22].

The predominant function of autophagy is to promote cell survival following stress/nutrient limitation by recycling essential cellular components [32, 33]. Indeed, blocking autophagy, either genetically or chemically, generally promotes the demise of cells under stress conditions [34]. However, in specific contexts, autophagy also leads to cell death $[10,12,15]$. With much of our understanding of the regulation and mechanism of autophagy from studies on its role in cell survival, the physiological role and the molecular mechanisms of autophagy-dependent cell death are less well characterised.

\section{Autophagy in cell death}

Early observations of dying cells reported the accumulation of autophagosomes and autolysosomes in the cytoplasm [35] and there have been many reports since prompting the use of the term 'autophagic cell death'. However, in many cases, the evidence of a causal role for autophagy was not established while other observations were based mainly on morphological characteristics. As discussed recently [14], the interplay between autophagy and various forms of cell death makes it difficult to distinguish the specific roles of autophagy in mediating cell death. As such the concept of cell death dependent entirely on autophagy has remained somewhat controversial.

Based on guidelines published by a large section of the scientific community [15, 36] the roles of autophagy in cell death can be defined as: (i) autophagy-associated cell death, where by the induction of autophagy coincides with the induction of apoptosis (or other cell death pathways), where autophagy simply accompanies the cell death process and does not have an active role in it; (ii) autophagy-mediated cell death, where autophagy induction triggers apoptosis; and (iii) autophagy-dependent cell death, a distinct mechanism of cell death that occurs independently of apoptosis or necrosis 

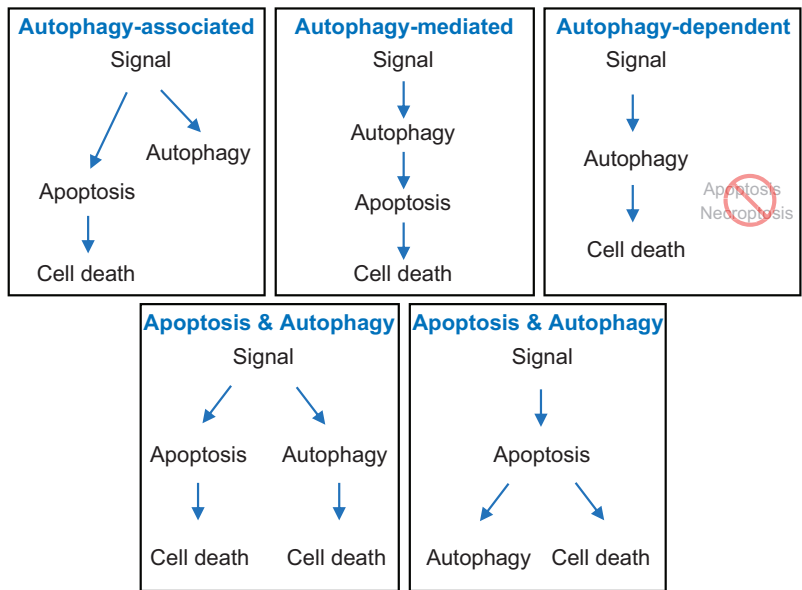

Fig. 1 Roles of autophagy in cell death. The role of autophagy in cell death can be: autophagy-associated cell death, where the induction of autophagy accompanies apoptosis (or other cell death pathways); autophagy-mediated cell death, where the autophagy pathway activates apoptosis (or other cell death modalities); and autophagydependent cell death, which occurs independently of apoptosis or necrosis (e.g. Drosophila larval midgut degradation). An additional context-specific mode of cell death involves the coordinated action of both apoptosis and autophagy in parallel (e.g. Drosophila salivary gland degradation [70]), and Bax- and Bak-mediated induction of both apoptosis and autophagy [116]

(Fig. 1). The Nomenclature Committee of Cell Death defines autophagy-dependent cell death as 'a form of regulated cell death that mechanistically depends on the autophagic machinery (or components thereof)' [10]. The autophagymediated cell death must strictly follow the criteria that the inhibition of autophagy, through either genetic or chemical means, prevents cell death [10, 15, 36, 37]. As several components of the autophagy machinery have autophagyindependent functions [38] it is recommended to establish the involvement of at least two different proteins of the autophagy machinery $[15,36]$. Additionally, in the case of autophagy-dependent cell death, even where inhibition of autophagy prevents cell death, evidence that apoptosis or necrosis are either not involved or function in parallel with these death modalities is also required.

\section{Examples of autophagy-dependent cell death}

Several Atg gene knockout (KO) mice have been generated that have provided important insights into the physiological functions of autophagy in vivo [39]. However, dissecting the contribution of autophagy to cell death using mice has proven difficult due to early lethality, other overt phenotypes or redundancy in Atg gene function [39, 40]. Studies in mammalian and other model systems have clearly identified instances of autophagy-dependent cell death in specific developmental or pathophysiological contexts. Thus, autophagy appears to have highly context-specific functions in cell death rather than a wider role, yet many aspects of the physiological roles of autophagy in vivo remain unclear.

A form of autophagy-dependent cell death, called 'autosis', involving the $\mathrm{Na}^{+} / \mathrm{K}^{+}$-ATPase occurs in cells following treatment with a BECN1-derived peptide (TatBeclin 1) or following starvation [41]. The dying cells show morphological characteristics of autophagy, not apoptosis or necrosis, and cell death can be prevented by knockdown of Atg13 or Atg14, or by chemically blocking autophagosome formation. The genetic or chemical inhibition of apoptosis or necrosis does not rescue Tat-Beclin 1-induced cell death. Autosis has been shown to occur in the brain of newborn rats following hypoxia-ischaemic injury [41, 42].

There is genetic evidence for autophagy-dependent cell death in mammalian cells that are unable to undergo apoptosis. Removal of interdigital web cells during embryonic development is delayed in apoptosis-deficient Bax/Bak KO mice and is further delayed in Atg5/Bax/Bak triple KO mice [43]. This suggests that autophagydependent cell death may be important to compensate when apoptosis is blocked or inhibited. There is further evidence for autophagy driving cell death in the absence of apoptosis. In Bax/Bak-deficient mouse embryonic fibroblasts, autophagy is required for cell death induced by chemotherapeutic drugs, which is blocked by genetically inhibiting autophagy [44]. This compensatory action of autophagy is also observed in both cultured and primary cell lines treated with caspase inhibitors or that also lack apoptosis machinery [43, 45, 46].

There are also examples in primary and transformed cells with functional apoptotic machinery where the induction of autophagy can lead to cell death. In human ovarian epithelial cancer cells, expression of oncogenic H-Ras induced autophagy resulting in cell death that was prevented by knockdown of Atg5 or Atg7 [47]. In fibroblast cell lines, the expression of oncogenic Ras induced caspase-independent cell death accompanied by accumulation of autophagosomes and upregulation of Atg5 [48]. Chemical inhibition of autophagy prevented Ras-induced cell death, indicating that autophagy is promoting cell death rather than survival in these cells [48]. However, in other studies using transformed cells, expression of activated Ras can promote cell growth accompanied by increased autophagy [49-51]. In addition to these examples, there have been many studies performed in cell culture; studies are needed in animal models of cancer to determine if induction of autophagy can lead to tumour cell death in vivo.

A recent model of autophagy-dependent cell death has been established using resveratrol treatment of A549 lung carcinoma cells [52]. An increase in autophagic flux and 


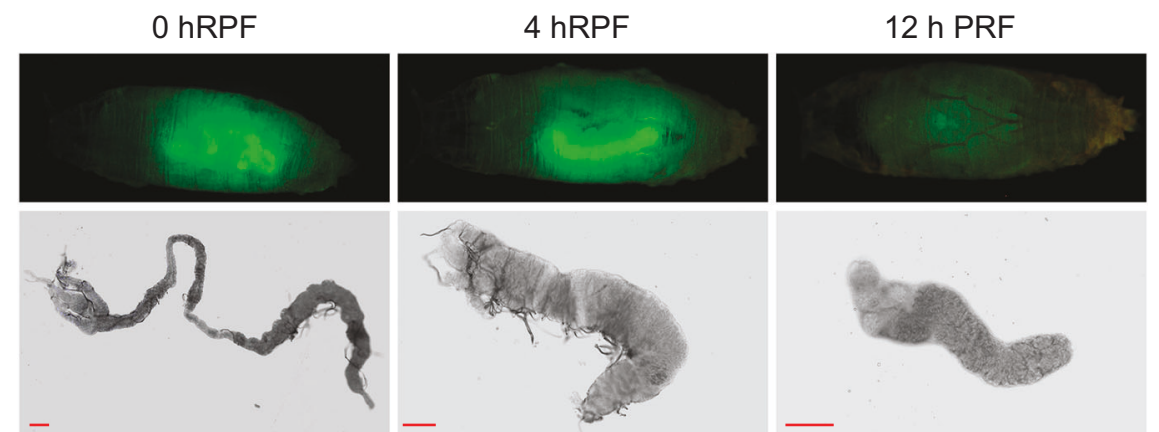

Fig. 2 Degradation of the Drosophila larval midgut. During metamorphosis, an increase in the steroid hormone ecdysone triggers the degradation of the larval midgut by autophagy-dependent cell death. Stages of midgut removal observed by GFP in whole animals, from the onset of metamorphosis at $0 \mathrm{~h}$ relative to puparium formation (hRPF)

cell death following resveratrol treatment occurs without activation of apoptosis and/or necroptosis. This cell death is associated with an increase in autophagic markers and could not be blocked by inhibiting apoptosis or necroptosis. Importantly, knockdown of several autophagy genes (ATG4B, ATG7, ATG12 and MAP1LC3B) decreased resveratrol-induced LC3 lipidation and increased cell viability [52]. However, depletion of ULK1 or BECN1 did not block cell death indicating that resveratrol-induced cell death is ULK1- and BECN1-independent. Using this model to perform a signalome-wide RNAi screen identified $G B A 1$, the Gaucher disease-associated gene, which encodes an enzyme that metabolises glucosylceramide to ceramide and glucose, as an essential factor mediating enhanced selfconsumption of intracellular components and autophagydependent cell death [52]. Interestingly, GBA1 was also found to be important for the developmental cell death in Drosophila larval midgut [53], a tissue that is well known to be removed by an autophagy-dependent process (see below).

The main mode of PCD in C. elegans is apoptosis, and autophagy can promote cell death when apoptosis is reduced. In the germline, autophagy can contribute to cell death in response to genotoxic stress or when apoptotic activity is reduced but does not appear to be essential under normal physiological conditions [54]. Depletion of autophagy gene function reduced germ cell corpses in ced-3 mutants or following irradiation or ethylnitrosourea treatment. This suggests that when caspase activity is partially compromised, autophagy can contribute to the execution of cell death.

Developmentally programmed induction of autophagy and apoptosis occurs during metamorphosis in holometabolous insects [55]. The early morphological identification of PCD from studies examining the removal of intersegmental muscles of silk moths showed features of autophagy [56]. In several Lepidoptera species, the degradation of during midgut degradation at $4 \mathrm{hRPF}$ and following midgut condensation at $12 \mathrm{hRPF}$. The degradation is characterised by the initial contraction of the gastric caeca followed by condensation of the midgut body (scale bar represents $200 \mu \mathrm{m}$ )

larval organs including the midgut, fat body and silk gland shows morphological features of both apoptosis and autophagy (similar to the Drosophila larval midgut and salivary glands). In Helicoverpa armigera, knockdown of Atg12 blocks larval midgut removal, suggesting that autophagy is required for cell death in this tissue [57]. However, in most cases, the genetic/molecular contribution of each pathway to cell death in the specific tissues remains to be established.

\section{Autophagy-dependent cell death in vivo}

A unique example of autophagy-dependent cell death is found in the amoeba Dictyostelium discoideum, that does not encode caspases or apoptotic machinery genes [58]. The transition from vegetative state to fruiting body requires PCD that is prevented by genetically blocking autophagy $[59,60]$. Initially autophagy is induced in response to starvation and following this, cell death is triggered in response to an additional signal, differentiation-inducing factor (DIF-1). Both signals are required to induce cell death as starvation alone or DIF-1 exposure to non-starved cells is not sufficient to induce death. It is intriguing that these signals combine to convert a survival function of autophagy to a cell death promoting role. This is an example of autophagy during cell death in the absence of apoptosis, whereas in higher organisms with intact apoptosis machinery, the complex relationship between autophagy and apoptosis (other modes of cell death) occurs.

The strongest evidence for the physiological role of autophagy as a cell death mechanism comes from developmentally PCD in Drosophila. While most developmental and stress-induced cell death in Drosophila is executed by apoptosis, autophagy-dependent cell death occurs in specific tissues [61]. During the larval-pupal transition, pulses of the steroid hormone ecdysone trigger the degradation of 
obsolete larval tissues including the midgut and salivary glands [62-64] (Fig. 2). Despite upregulation of apoptosis genes and high levels of caspase activity, degradation of the larval midgut proceeds normally in the absence of the apoptotic machinery or inhibition of caspase activity [65]. However, depletion of particular components of the autophagy machinery delays midgut degradation [65-67]. Furthermore, the combined inhibition of both apoptosis and autophagy does not further delay midgut degradation than inhibition of autophagy [65].

Similar to the larval midgut, salivary gland degradation exhibits high levels of autophagy and caspases [64, 68, 69]. However, unlike midgut degradation, the removal of the salivary glands requires both apoptosis and autophagy in parallel for their degradation [70]. The inhibition of either apoptosis and autophagy alone results in partial salivary gland removal, whereas the combined inhibition of both pathways further delays their degradation, suggesting that these pathways act in parallel [70]. Thus, salivary gland PCD can be considered a unique example where both apoptosis and autophagy-dependent cell death act in parallel. These obsolete tissues that are triggered to undergo PCD in response to ecdysone highlight the context-specific role of autophagy and the complex relationship between the apoptotic and autophagy pathways.

\section{How cells die by the autophagy-dependent process?}

Except for autosis, which is dependent on plasma membrane $\mathrm{Na}^{+} / \mathrm{K}^{+}$-ATPase [41], how cells die by autophagydependent cell death remains largely unknown. Ultrastructural analysis of cells dying by autophagy has provided some insights. In mammalian cells, ultrastructural and functional analyses indicate that most organelles, including mitochondria and the ER, appear normal during autophagydependent cell death [43]. Compared to cells undergoing starvation-induced autophagy, those undergoing cell death have increased number and size of autophagic vacuoles/ vesicles [43], suggesting that hyperactivation of autophagy may promote cell death. Indeed, cell death is enhanced when feedback mechanisms to restrain autophagy are disrupted [71]. Similarly, degraded mitochondria, intracellular membranes and cytoplasmic material could be detected in cells dying by autophagy [52]. This supports the idea that excessive consumption of cytoplasmic components upon sustained autophagy leads to cell death. In Drosophila, blocking autophagy-dependent cell death in the midgut leads to the accumulation of mitochondria suggesting an important role for the bulk clearance of cytoplasmic material [66]. While autophagy is required for mitochondrial clearance in the midgut, it is yet to be determined whether

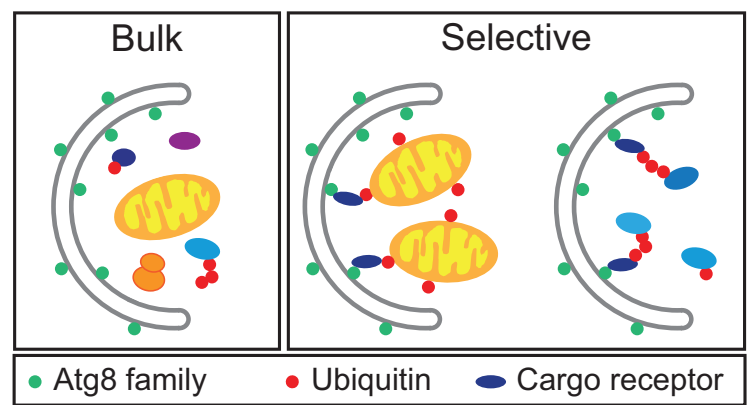

Fig. 3 Possible targets of autophagy during cell death. The cellular targets engulfed during autophagy-dependent cell death remain to be determined. Examples of possible targets include the bulk engulfment of cytoplasmic contents including cytosol and organelles or forms of selective autophagy that promote the clearance of mitochondria (e.g. mitophagy) or ubiquitinated proteins (e.g. aggrephagy)

mitochondria are preferentially degraded over other cellular components [66, 72]. During midgut degradation, the Drosophila homologue of mammalian autophagy receptor p62, refractory to sigma $\mathrm{P}(\operatorname{ref}(2) \mathrm{P})$, is not essential for autophagy, suggesting that cargo selection may be important in determining whether non-selective bulk degradation of cytoplasmic targets is required or if specific cargoes are selected [67, 72]. However, whether selective autophagy contributes to autophagy-dependent midgut PCD is unknown (Fig. 3).

Autophagic flux is dependent on lysosomal function, and it is likely that the lysosome will contribute to autophagydependent cell death. The degradation of larval midgut cells that are deficient for $M a d$ and $t k v$ has revealed unique features of autophagy-dependent cell death. The Mad and tkv mutant cells are rapidly degraded with markers of autophagy and lysosome activity suggesting that the demise of the cell is due to the bulk degradation of cellular components, presumably by the lysosomal enzymes [73]. Another recent study suggests that the lysosome may contribute to cell death due to autophagy [74]. Following prolonged starvation, Bax/Bakl double KO Mouse Embryonic Fibroblasts (MEFs) are resistant to autophagydependent cell death. Targeting Bax (or a monomeric-only mutant form of Bax) to the lysosome (or treatment with a lysomotropic detergent) increased lysosomal membrane permeability and restored starvation-induced autophagic cell death in Bax/Bakl double KO MEFs [74]. However, it remains to be determined if the degradation of specific organelles initiates autophagy-dependent cell death or if the bulk degradation of cytosolic components by autophagy is the primary cause of cell death. The contribution of the lysosome to autophagy-dependent cell death also warrants further investigation.

Another mechanism that has been proposed is that autophagy may selectively degrade specific survival factors to induce cell death. For example, the Drosophila inhibitor 
of apoptosis protein dBruce localises to autophagosomes and is necessary for caspase-dependent cell death of the ovary [75]. The developmental cell death in the oocyte is triggered by the autophagy-mediated degradation of the anti-apoptotic dBruce that enables the subsequent activation of caspase-dependent apoptosis.

\section{The autophagy machinery in cell death}

The autophagy machinery required to mediate cell death may differ to that promoting cell survival. There may be differences in the rate of autophagic flux, the length of time the pathway is active and whether the engulfed contents are being recycled or degraded. All these factors may contribute to distinct requirements for autophagy machinery components. The genetic dissection of the requirement of the Atg genes to autophagy-dependent Drosophila midgut degradation revealed that only a subset of the individual components are required (Table 1). In the Drosophila midgut, RNAi-mediated screening of the Atg machinery revealed

Table 1 Components of the autophagy pathway that are essential for midgut degradation compared to starvation-induced autophagy in the fat body

\begin{tabular}{|c|c|c|c|}
\hline & $\begin{array}{l}\text { Drosophila Atg } \\
\text { gene }\end{array}$ & $\begin{array}{l}\text { Midgut } \\
\text { degradation }\end{array}$ & $\begin{array}{l}\text { Starvation in } \\
\text { fat body }\end{array}$ \\
\hline \multirow[t]{4}{*}{ Initiation } & Atg1 & $\checkmark$ & $\checkmark$ \\
\hline & $\operatorname{Atg} 13$ & $\checkmark$ & $\checkmark$ \\
\hline & $\operatorname{Atg} 17$ & $\checkmark$ & $\checkmark$ \\
\hline & Atg101 & $\checkmark$ & $?$ \\
\hline \multirow[t]{4}{*}{ Nucleation } & Atg6 & $x$ & $\checkmark$ \\
\hline & Atg14 & $x$ & $\checkmark$ \\
\hline & Vps15/ird1 & $\checkmark$ & $\checkmark$ \\
\hline & $\begin{array}{l}\text { Vps34/ } \\
\text { Pi3K59F }\end{array}$ & $\checkmark$ & $\checkmark$ \\
\hline \multirow{10}{*}{$\begin{array}{l}\text { Conjugation } \\
\text { systems }\end{array}$} & Atg3/Aut1 & $x$ & $\checkmark$ \\
\hline & $\operatorname{Atg} 4 a$ & $x$ & $\checkmark$ \\
\hline & $\operatorname{Atg} 4 b$ & $?$ & $?$ \\
\hline & Atg5 & $x$ & $\checkmark$ \\
\hline & $\operatorname{Atg} 7$ & $x$ & $\checkmark$ \\
\hline & Atg8a & $\checkmark$ & $\checkmark$ \\
\hline & $\operatorname{Atg} 8 b$ & $?$ & $?$ \\
\hline & $\operatorname{Atg} 10$ & $\checkmark$ & $?$ \\
\hline & $\operatorname{Atg} 12$ & $x$ & $\checkmark$ \\
\hline & $\operatorname{Atg} 16$ & $x$ & $\checkmark$ \\
\hline \multirow{4}{*}{$\begin{array}{l}\text { Atg9 trafficking } \\
\text { system }\end{array}$} & Atg9 & $\checkmark$ & $\checkmark$ \\
\hline & Atg2 & $\checkmark$ & $\checkmark$ \\
\hline & $\operatorname{Atg} 18 \mathrm{a}$ & $\checkmark$ & $\checkmark$ \\
\hline & Atg18b & $?$ & $?$ \\
\hline
\end{tabular}

$\checkmark$ Required; $\boldsymbol{x}$ Not essential; ? Unknown that a subset of the multi-subunit complexes that are required for starvation-induced autophagy in the fat body are required for autophagy-dependent cell death in the midgut [67]. The components of the initiation complex (Atg1, Atg13, Atg17 and Atg101) and PtdIns(3)P binding proteins (Atg9, Atg2 and Atg18), as well as specific components of class III PI3K complex (Vps15 and Vps34), and Atg8a are required for autophagy-dependent midgut degradation [67]. However, components of the two conjugation systems, including Atg7 (the E1 enzyme) and Atg3 (the E2 enzyme), are not required for this process although these genes are essential for starvation-induced autophagy in the fat body $[66,67]$. Autophagy-dependent cell death in the Drosophila larval midgut appears to be regulated differently from autophagy in cell survival context. These findings highlight the cell type- and context-specific differences in the utilisation of autophagic machinery. This may contribute to altered rates of autophagy flux or to maintain the activity of the pathway for an extended time.

There is increasing evidence that a number, if not all, of Atg proteins have functions in addition to their role in autophagy [38]. An autophagy-independent function of Atg5-Atg12/Atg16L1 protein complex has been described in the antiviral activity of interferon gamma [76]. Individual autophagy pathway components also play roles in cell death that are independent of their role in autophagy [77, 78]. Following genotoxic stress, the increase in ATG5 expression in the nucleus promotes mitotic catastrophe [79]. The conjugation of Atg12 to Atg3 during intrinsic mitochondrial-mediated apoptosis does not occur during starvation-induced autophagy [80]. Further characterisation of autophagy-dependent or -independent functions of a particular Atg protein will aid in distinguishing the contributions to various biological processes including cell death.

The ubiquitin system has multiple roles in the regulation of autophagy [81, 82]. Many components of the autophagy machinery are modified by ubiquitin to regulate autophagic flux, from initiation to termination. Ubiquitination is also important for targeting of cargoes in several forms of selective autophagy [83]. Cargo receptors bind to specific ubiquitinated substrates to direct the selective incorporation of the cargo into autophagosomes. In Drosophila, ubiquitination is important for tissue-specific autophagy-dependent cell death. Ubiquitination is essential for midgut autophagy and cell size reduction that accompanies midgut degradation [66]. As described above, the components of the conjugation machinery, Atg7 and Atg3, are not required for autophagy-dependent midgut degradation. To identify the putative E1-activating enzymes (that function in place of Atg7), an RNAi-mediated screen in Drosophila larval midgut identified Ubal as a specific regulator of autophagy and cell size reduction during midgut cell death [66]. 
The function of Uba1 is downstream of Atg1 but does not replace Atg7. This raises the possibility that additional components of the ubiquitin system (e.g. E2s, E3s) may be necessary for autophagy-dependent cell death of the midgut.

\section{Signals regulating autophagy-dependent cell death}

The different forms of autophagy appear to require distinct regulatory components. Given the highly context-specific requirement for autophagy-dependent cell death, there may be specific signals to initiate the cell death function of autophagy.

\section{Ecdysone-dependent signalling during metamorphosis}

As mentioned above, the steroid hormone ecdysone plays an important role in triggering the degradation of the obsolete larval midgut and salivary gland in Drosophila $[62,64,69]$. Increased levels of ecdysone bind to the heterodimeric nuclear receptor complex, ecdysone receptor (EcR) and ultraspiracle (USP) in a spatio-temporal manner to regulate the expression of genes involved in proliferation, differentiation and PCD [84-87]. Following puparium formation, the rise in ecdysone levels triggers expression of genes regulating both apoptosis and autophagy resulting in salivary gland cell death $[68,69,88]$. The binding of EcR/ Usp recruits histone-modifying enzymes including the histone demethylase dUTX to the promoters to activate the expression of a number of key apoptosis and autophagy genes during salivary gland PCD [89-92]. Coincident with the increase in ecdysone during midgut degradation, many Atg genes are transcriptionally upregulated immediately prior to larval midgut degradation in an EcR-dependent manner [73]. The knockdown of $E c R$ in the midgut blocks the increase in Atg genes preventing autophagy induction and delaying midgut degradation [73]. Despite the upregulation of Atg gene expression in response to ecdysone, the direct transcriptional regulators responsible still remain to be identified.

The role of ecdysone in regulating the expression of autophagy genes appears to be conserved in other insects. In Lepidoptera (silk worm), the induction of autophagy and apoptosis gene expression during metamorphosis occurs in response to ecdysone 20E [93]. In other Diptera (sand flies), the upregulation of Atg gene expression coincides with increased ecdysone during midgut removal; however, it is yet to be established if they are regulated by ecdysone [94]. There may be additional roles for ecdysone in the regulation of autophagy pathway activity as in $H$. armigera conjugation of ATG12-ATG5 occurs in response to ecdysone in a concentration- and time-dependent manner [57].

\section{Growth signalling}

Induction of autophagy under conditions of nutrient limitation and growth factor withdrawal generally promotes cell survival. A central regulator coordinating autophagy to nutrients and growth factors is the mechanistic target of rapamycin (mTOR) kinase [95]. The function of mTOR is dependent on two distinct complexes distinguished by unique components with TOR complex 1 (TORC1) central in regulating autophagy containing Raptor (regulatory associated protein of TOR), and TOR complex 2 (TORC2) containing Rictor (rapamycin insensitive companion of TOR) [96]. In the presence of nutrients and growth signals, TORC1 activity prevents autophagy by phosphorylating and inhibiting Atg1/ULK1 complex activity [97]. In growth-limiting conditions such as starvation, TORC1 is no longer active enabling Atg1 activation and the initiation of autophagy [98].

During Drosophila larval development, mTOR is required for cellular growth in the salivary glands and midgut. Similar to conditions of nutrient limitation where TORC1 inactivation results in the induction of autophagy, inactivation of TORC1 is required for autophagy-dependent midgut cell death [67]. The knockdown of Tor and raptor (but not TORC2 component rictor) induced premature autophagy and midgut degradation [67]. Thus, TORC1 inactivation is required for the induction of autophagy in both cell survival and cell death. This suggests that while the molecular machinery mediating autophagy-dependent cell death is distinct to that required for cell survival, the initiation of autophagy may occur similarly.

Several growth signalling pathways, including the insulin receptor/class I phosphoinositide-3-kinase (PI3K) pathway and receptor tyrosine kinase via Ras/mitogen-activated protein kinase (MAPK) pathway, can promote the activation of TORC1 resulting in the inhibition of autophagy $[96,98]$. It also appears that these same pathways can also regulate autophagy in the context of cell death. In Drosophila, the maintenance of growth signalling by class I PI3K pathway blocks autophagy induction preventing the degradation of larval salivary gland and midgut tissue during metamorphosis [70, 99]. The downregulation of PI3K activity by the expression of PTEN, TSC1 and TSC2 was sufficient to induce premature induction of autophagy and midgut degradation [99]. Thus, the developmental cell death of the fat body, salivary glands and midgut all require autophagy and downregulation of PI3K signalling [70, 99, 100]. This suggests that despite the opposing roles of autophagy in cell survival and cell death, the induction of autophagy occurs in response to similar signals. The 
upstream signals that regulate the induction of autophagy including growth arrest and inactivation of TORC1 are similar; however, the molecular machinery mediating cell death may be distinct.

As described above, oncogenic Ras has a complex relationship between autophagy, cell growth and cell death. Depending on the context, activated Ras can induce autophagy promoting cell growth and survival or, under different conditions, it can result in cell death due to increased autophagy [47, 49]. In mammals, caspase-independent $\mathrm{H}$-Ras mediated cell death requires the BH3-only protein Noxa and Beclin-1, with knockdown of Noxa or Beclin-1 reducing autophagy and promoting survival. In the presence of Noxa, Beclin-1 dissociates from anti-apoptotic protein Mcl-1, promoting autophagy induction. This indicates that in response to oncogenic Ras, Noxa may participate in the activation of the autophagy machinery to promote cell death by a unique caspase-independent mechanism [47]. However, the expression of Ras in the Drosophila larval salivary gland and midgut tissue maintains growth signalling and blocks autophagy induction preventing tissue degradation [70, 99].

\section{Decapentaplegic signalling}

In recent studies, the Drosophila bone morphogenetic protein/transforming growth factor $\beta$ ligand BMP/TGF- $\beta$, Decapentaplegic (Dpp), was identified as a suppressor of autophagy-dependent midgut degradation [73]. In the absence of Dpp signalling premature autophagy induction and rapid cell death occurred in midgut cells, whereas maintaining Dpp signalling inhibited autophagy induction [73]. While Dpp plays an important role in the timing of autophagy-dependent midgut degradation, other key morphogens $\mathrm{Hh}$ and $\mathrm{Wg}$ are not required for autophagydependent cell death [101]. As sustained Dpp signalling in the midgut prevents the expression of ecdysone responsive genes and impairs ecdysone production, Dpp may act in inter-organ communication to coordinate ecdysone biosynthesis and timing of autophagy-dependent midgut degradation [73]. In mammals, members of the TGF- $\beta$ superfamily induce apoptosis and autophagy in vitro in some primary and transformed cell lines [102-104]. However, the physiological role of TGF- $\beta$ signalling in autophagy-dependent cell death in mammals remains to be established.

\section{Other genes/pathways regulating autophagy- dependent cell death}

The discovery of additional genes that function during autophagy in specific contexts suggests that the canonical autophagy machinery may not be the same in all cells or tissues. The requirements for the degradation of the larval salivary glands compared to the larval midgut are distinct, despite apparent similarity in the requirement of autophagy for the degradation of these tissues. For instance, the Hippo pathway is required for the correct removal of salivary glands but not for midgut cell death [99, 105].

A number of genes have a context-specific role in the regulation of autophagy during salivary gland degradation [106-110]. The engulfment receptor Draper (Drpr, MEGF10 in mammals) is required for autophagy upstream of Atg1 for PCD of the salivary glands [106]. Draper is present on the surface of salivary gland cells and acts in a cell autonomous manner to promote degradation and clearance of the tissue [106]. In addition, the non-cell autonomous function of Macroglobulin complement-related (Mcr) is required for the regulation of autophagy that is dependent on Drpr in neighbouring cells [107]. In salivary glands, miR-14 and calcium signalling regulate autophagy and cell death [110]. The miR-14 targets inositol 1,4,5-triphosphate kinase 2 (ip3k2), regulates inositol 1,4,5-trisphosphate (IP3) signalling and release of calcium from the ER [110]. This subsequently affects the activity of the calcium-binding messenger protein Calmodulin. The Ras-like protein A (Ral), a Ras superfamily small GTPases, and the exocyst components have a context-specific role in autophagy during salivary gland PCD [109]. In mammals, Ral regulates stress-induced autophagy by interacting with the components of the exocyst [111]. However, Ral is dispensable for starvation-induced autophagy in the Drosophila fat body [109]. A role for dynein light chain 1 (ddlc1) in protein clearance by autophagy is required for salivary gland degradation [108]. The proton-coupled pyruvate transporter, Hermes, acts as a negative regulator of mTOR signalling required for autophagy during salivary gland cell death [112]. The roles of these genes in salivary gland PCD may reflect the requirement of the cell death pathways in this tissue and highlights the importance of investigating regulatory mechanisms in a context-specific manner.

Tissue-specific regulators of autophagy for cell death during midgut degradation, and not during salivary gland degradation or starvation-induced autophagy, have also been identified. As described above, the E1, Uba1, was found to be necessary for midgut cell death, but not for salivary gland cell death [66]. This raised the possibility that additional components of the ubiquitin-conjugation system such as E2s and E3s may regulate autophagy-dependent cell death of the midgut. The ubiquitin-binding UBA domain containing protein, $\mathrm{Vps} 13 \mathrm{D}$ is a specific regulator of autophagy-dependent cell death in the midgut and does not affect starvation or stress-induced autophagy [72]. Knockdown of Vps13D or deletion of the UBA domain prevents the clearance of mitochondria during midgut degradation, a phenotype similar to blocking autophagy, with enlarged 
mitochondria $[66,72]$. The function of VPS13D in the regulation of mitochondrial morphology appears to be conserved as VPS13D mutant human cells also have more large and rounded mitochondria. However, it remains to be established if Vps13D also functions in autophagydependent cell death in the larval midgut and if such a function is conserved in cell death in mammals.

The receptor tyrosine phosphatase 52F (PTP52F) is required for midgut degradation. The knockdown of PTP52F delayed midgut degradation that could be rescued by the expression of the ATPase VCP (valosin-containing protein) orthologue Ter94 [113]. VCP participates in many cellular processes including cell cycle regulation and ubiquitin-proteasome system [114], as well as in several steps of the autophagy pathway, including autophagosome maturation, autophagosome-lysosome fusion, and is required for the clearance of damaged lysosomes by autophagy [115]. This suggests that Ter94 may have other roles or may play a more general role in autophagy. Whether PTP52F function is limited to autophagy-dependent cell death or if it also plays a role in the regulation of starvation-induced autophagy for cell survival remains to be determined.

To identify novel regulators in mammalian cells, a screen using resveratrol-induced autophagy-dependent cell death of lung carcinoma identified GBA1, a glucocerebrosidase enzyme that converts glucosylceramide to glucose and ceramide in the lysosome [52]. The knockdown of GBAI blocked resveratrol-induced autophagy-dependent cell death and reduced ceramide production. The function of GBA1 in autophagy-dependent cell death is evolutionarily conserved in Drosophila with Gbala knockdown delaying midgut cell death $[52,53]$. However, it remains to be determined how GBA1/Gba1a activate autophagydependent cell death.

\section{Future perspectives}

As described above, the function of autophagy in promoting cell death is context dependent. The signals and molecular machinery that distinguish autophagy-dependent cell death to that of autophagy required for cell survival is only beginning to be elucidated. There is evidence that the autophagy machinery required during cell death is distinct to the machinery required during cell survival, but it remains to be established how conserved this is and what additional components function in cell death (and if they are cell specific). The induction of autophagy occurs in response to multiple signals and several of the key regulators are similar in both surviving and dying cells. There are also distinct regulatory mechanisms of autophagydependent cell death that act in specific cell/tissue types, and how these signals are integrated may dictate the cellular outcomes. Whether the internal/external cues promote a signal to increase the rate of flux or prolong autophagy (or prevent termination) resulting in the demise of the cell remains to be established. The molecular mechanisms that lead to the demise of the cell during autophagy-dependent cell death are poorly understood. There are a number of specific forms of autophagy that are defined based on the cargo selected for degradation, and whether autophagydependent cell death involves the degradation of selective cargo or bulk degradation of cytoplasmic components remains to be established. Given the context-specific role and distinct molecular machinery mediating autophagydependent cell death, it will be important to investigate the regulatory mechanisms in the physiological settings.

Acknowledgements This work was supported by the National Health and Medical Research Council (NHMRC) of Australia Project Grant (1124490), an Australian Research Council Discovery Grant (DP170100623) and an NHMRC Senior Principal Research Fellowship (1103006).

\section{Compliance with ethical standards}

Conflict of interest The authors declare that they have no conflict of interest.

\section{References}

1. Yuan J, Kroemer G. Alternative cell death mechanisms in development and beyond. Genes Dev. 2010;24:2592-602.

2. Ellis RE, Yuan JY, Horvitz HR. Mechanisms and functions of cell death. Annu Rev Cell Biol. 1991;7:663-98.

3. Cashio P, Lee TV, Bergmann A. Genetic control of programmed cell death in Drosophila melanogaster. Semin Cell Dev Biol. 2005;16:225-35.

4. White K, Grether ME, Abrams JM, Young L, Farrell K, Steller H. Genetic control of programmed cell death in Drosophila. Science. 1994;264:677-83.

5. Ellis HM, Horvitz HR. Genetic control of programmed cell death in the nematode C. elegans. Cell. 1986;44:817-29.

6. Yuan J, Shaham S, Ledoux S, Ellis HM, Horvitz HR. The $C$. elegans cell death gene ced-3 encodes a protein similar to mammalian interleukin-1 beta-converting enzyme. Cell. 1993; 75:641-52.

7. Knudson CM, Tung KS, Tourtellotte WG, Brown GA, Korsmeyer SJ. Bax-deficient mice with lymphoid hyperplasia and male germ cell death. Science. 1995;270:96-9.

8. Lindsten T, Ross AJ, King A, Zong WX, Rathmell JC, Shiels $\mathrm{HA}$, et al. The combined functions of proapoptotic Bcl-2 family members bak and bax are essential for normal development of multiple tissues. Mol Cell. 2000;6:1389-99.

9. Chautan M, Chazal G, Cecconi F, Gruss P, Golstein P. Interdigital cell death can occur through a necrotic and caspaseindependent pathway. Curr Biol. 1999;9:967-70.

10. Galluzzi L, Vitale I, Aaronson SA, Abrams JM, Adam D, Agostinis P, et al. Molecular mechanisms of cell death: recommendations of the Nomenclature Committee on Cell Death 2018 Cell Death Differ. 2018;25:486-541. 
11. Shalini S, Dorstyn L, Dawar S, Kumar S. Old, new and emerging functions of caspases. Cell Death Differ. 2015;22:526-39.

12. Denton D, Nicolson S, Kumar S. Cell death by autophagy: facts and apparent artefacts. Cell Death Differ. 2012;19:87-95.

13. Anding AL, Baehrecke EH. Autophagy in cell life and cell death. Curr Top Dev Biol. 2015;114:67-91.

14. Doherty J, Baehrecke EH. Life, death and autophagy. Nat Cell Biol. 2018;20:1110-7.

15. Galluzzi L, Baehrecke EH, Ballabio A, Boya P, Bravo-San Pedro JM, Cecconi F, et al. Molecular definitions of autophagy and related processes. EMBO J. 2017;36:1811-36.

16. Li WW, Li J, Bao JK. Microautophagy: lesser-known self-eating. Cell Mol Life Sci. 2012;69:1125-36.

17. Kaushik S, Cuervo AM. Chaperone-mediated autophagy: a unique way to enter the lysosome world. Trends Cell Biol. 2012;22:407-17.

18. Tsukada M, Ohsumi Y. Isolation and characterization of autophagy-defective mutants of Saccharomyces cerevisiae. FEBS Lett. 1993;333:169-74.

19. Thumm M, Egner R, Koch B, Schlumpberger M, Straub M, Veenhuis $\mathrm{M}$, et al. Isolation of autophagocytosis mutants of Saccharomyces cerevisiae. FEBS Lett. 1994;349:275-80.

20. Harding TM, Morano KA, Scott SV, Klionsky DJ. Isolation and characterization of yeast mutants in the cytoplasm to vacuole protein targeting pathway. J Cell Biol. 1995;131:591-602.

21. Mizushima N, Komatsu M. Autophagy: renovation of cells and tissues. Cell. 2011;147:728-41.

22. Yu L, Chen Y, Tooze SA. Autophagy pathway: cellular and molecular mechanisms. Autophagy. 2018;14:207-15.

23. Noda NN, Inagaki F. Mechanisms of autophagy. Annu Rev Biophys. 2015;44:101-22.

24. Antonioli M, Di Rienzo M, Piacentini M, Fimia GM. Emerging mechanisms in initiating and terminating autophagy. Trends Biochem Sci. 2017;42:28-41.

25. Yin Z, Pascual C, Klionsky DJ. Autophagy: machinery and regulation. Microb Cell. 2016;3:588-96.

26. Mizushima N. The role of the Atg1/ULK1 complex in autophagy regulation. Curr Opin Cell Biol. 2010;22:132-9.

27. Papinski D, Kraft C. Regulation of autophagy by signaling through the Atg1/ULK1 complex. J Mol Biol. 2016; 428:1725-41.

28. Russell RC, Tian Y, Yuan H, Park HW, Chang YY, Kim J, et al. ULK1 induces autophagy by phosphorylating Beclin-1 and activating VPS34 lipid kinase. Nat Cell Biol. 2013;15:741-50.

29. Geng J, Klionsky DJ. The Atg8 and Atg12 ubiquitin-like conjugation systems in macroautophagy. 'Protein modifications: beyond the usual suspects' review series. EMBO Rep. 2008; 9:859-64.

30. Mizushima N, Noda T, Yoshimori T, Tanaka Y, Ishii T, George $\mathrm{MD}$, et al. A protein conjugation system essential for autophagy. Nature. 1998;395:395-8.

31. Ohsumi Y. Molecular dissection of autophagy: two ubiquitinlike systems. Nat Rev Mol Cell Biol. 2001;2:211-6.

32. Kroemer G, Marino G, Levine B. Autophagy and the integrated stress response. Mol Cell. 2010;40:280-93.

33. Lum JJ, DeBerardinis RJ, Thompson CB. Autophagy in metazoans: cell survival in the land of plenty. Nat Rev Mol Cell Biol. 2005;6:439-48.

34. Boya P, Gonzalez-Polo RA, Casares N, Perfettini JL, Dessen P, Larochette $\mathrm{N}$, et al. Inhibition of macroautophagy triggers apoptosis. Mol Cell Biol. 2005;25:1025-40.

35. Schweichel JU, Merker HJ. The morphology of various types of cell death in prenatal tissues. Teratology. 1973;7:253-66.

36. Klionsky DJ, Abdelmohsen K, Abe A, Abedin MJ, Abeliovich $\mathrm{H}$, Acevedo Arozena A, et al. Guidelines for the use and interpretation of assays for monitoring autophagy (3rd edition). Autophagy. 2016;12:1-222.

37. Denton D, Xu T, Kumar S. Autophagy as a pro-death pathway. Immunol Cell Biol. 2015;93:35-42.

38. Cadwell K, Debnath J. Beyond self-eating: the control of nonautophagic functions and signaling pathways by autophagyrelated proteins. J Cell Biol. 2018;217:813-22.

39. Kuma A, Komatsu M, Mizushima N. Autophagy-monitoring and autophagy-deficient mice. Autophagy. 2017;13:1619-28.

40. Cecconi F, Levine B. The role of autophagy in mammalian development: cell makeover rather than cell death. Dev Cell. 2008;15:344-57.

41. Liu Y, Shoji-Kawata S, Sumpter RM Jr., Wei Y, Ginet V, Zhang L, et al. Autosis is a $\mathrm{Na}+, \mathrm{K}+$-ATPase-regulated form of cell death triggered by autophagy-inducing peptides, starvation, and hypoxia-ischemia. Proc Natl Acad Sci USA. 2013;110:20364-71.

42. Xie C, Ginet V, Sun Y, Koike M, Zhou K, Li T, et al. Neuroprotection by selective neuronal deletion of Atg7 in neonatal brain injury. Autophagy. 2016;12:410-23.

43. Arakawa S, Tsujioka M, Yoshida T, Tajima-Sakurai H, Nishida Y, Matsuoka Y, et al. Role of Atg5-dependent cell death in the embryonic development of Bax/Bak double-knockout mice. Cell Death Differ. 2017;24:1598-608.

44. Shimizu S, Kanaseki T, Mizushima N, Mizuta T, ArakawaKobayashi S, Thompson CB, et al. Role of Bcl-2 family proteins in a non-apoptotic programmed cell death dependent on autophagy genes. Nat Cell Biol. 2004;6:1221-8.

45. Lamy L, Ngo VN, Emre NC, Shaffer AL 3rd, Yang Y, Tian E, et al. Control of autophagic cell death by caspase-10 in multiple myeloma. Cancer Cell. 2013;23:435-49.

46. Yu L, Alva A, Su H, Dutt P, Freundt E, Welsh S, et al. Regulation of an ATG7-beclin 1 program of autophagic cell death by caspase-8. Science. 2004;304:1500-2.

47. Elgendy M, Sheridan C, Brumatti G, Martin SJ. Oncogenic Ras-induced expression of Noxa and Beclin-1 promotes autophagic cell death and limits clonogenic survival. Mol Cell. 2011;42:23-35.

48. Byun JY, Yoon CH, An S, Park IC, Kang CM, Kim MJ, et al. The Rac1/MKK7/JNK pathway signals upregulation of Atg5 and subsequent autophagic cell death in response to oncogenic Ras. Carcinogenesis. 2009;30:1880-8.

49. Guo JY, Chen HY, Mathew R, Fan J, Strohecker AM, KarsliUzunbas G, et al. Activated Ras requires autophagy to maintain oxidative metabolism and tumorigenesis. Genes Dev. 2011; 25:460-70.

50. Yang S, Wang X, Contino G, Liesa M, Sahin E, Ying H, et al. Pancreatic cancers require autophagy for tumor growth. Genes Dev. 2011;25:717-29.

51. Lock R, Roy S, Kenific CM, Su JS, Salas E, Ronen SM, et al. Autophagy facilitates glycolysis during Ras-mediated oncogenic transformation. Mol Biol Cell. 2011;22:165-78.

52. Dasari SK, Bialik S, Levin-Zaidman S, Levin-Salomon V, Merrill AH Jr., Futerman AH, et al. Signalome-wide RNAi screen identifies GBA1 as a positive mediator of autophagic cell death. Cell Death Differ. 2017;24:1288-302.

53. Dasari SK, Schejter E, Bialik S, Shkedy A, Levin-Salomon V, Levin-Zaidman $S$, et al. Death by over-eating: The Gaucher disease associated gene GBA1, identified in a screen for mediators of autophagic cell death, is necessary for developmental cell death in Drosophila midgut. Cell Cycle. 2017; 16:2003-10.

54. Wang H, Lu Q, Cheng S, Wang X, Zhang H. Autophagy activity contributes to programmed cell death in Caenorhabditis elegans. Autophagy. 2013;9:1975-82. 
55. Romanelli D, Casati B, Franzetti E, Tettamanti G. A molecular view of autophagy in Lepidoptera. Biomed Res Int. 2014; 2014:902315.

56. Lockshin RA, Williams CM. Programmed cell death-I. Cytology of degeneration in the intersegmental muscles of the Pernyi silkmoth. J Insect Physiol. 1965;11:123-33.

57. Li YB, Yang T, Wang JX, Zhao XF. The steroid hormone 20hydroxyecdysone regulates the conjugation of autophagy-related proteins 12 and 5 in a concentration and time-dependent manner to promote insect midgut programmed cell death. Front Endocrinol (Lausanne). 2018;9:28.

58. Cornillon S, Foa C, Davoust J, Buonavista N, Gross JD, Golstein P. Programmed cell death in Dictyostelium. J Cell Sci. 1994;107 (Pt 10):2691-704.

59. Giusti C, Tresse E, Luciani MF, Golstein P. Autophagic cell death: analysis in Dictyostelium. Biochim Biophys Acta. 2009; 1793:1422-31.

60. Luciani MF, Giusti C, Harms B, Oshima Y, Kikuchi H, Kubohara Y, et al. Atg1 allows second-signaled autophagic cell death in Dictyostelium. Autophagy. 2011;7:501-8.

61. Denton D, Aung-Htut MT, Kumar S. Developmentally programmed cell death in Drosophila. Biochim Biophys Acta. 2013;833:3499-506.

62. Jiang C, Baehrecke EH, Thummel CS. Steroid regulated programmed cell death during Drosophila metamorphosis. Development. 1997;124:4673-83.

63. Lee CY, Cooksey BA, Baehrecke EH. Steroid regulation of midgut cell death during Drosophila development. Dev Biol. 2002;250:101-11.

64. Lee CY, Simon CR, Woodard CT, Baehrecke EH. Genetic mechanism for the stage- and tissue-specific regulation of steroid triggered programmed cell death in Drosophila. Dev Biol. 2002; 252:138-48.

65. Denton D, Shravage B, Simin R, Mills K, Berry DL, Baehrecke $\mathrm{EH}$, et al. Autophagy, not apoptosis, is essential for midgut cell death in Drosophila. Curr Biol. 2009;19:1741-6.

66. Chang T-K, Shravage BV, Hayes SD, Powers CM, Simin RT, Wade Harper J, et al. Ubal functions in Atg7- and Atg3independent autophagy. Nat Cell Biol. 2013;15:1067-78.

67. Xu T, Nicolson S, Denton D, Kumar S. Distinct requirements of autophagy-related genes in programmed cell death. Cell Death Differ. 2015;22:1792-802.

68. Lee CY, Clough EA, Yellon P, Teslovich TM, Stephan DA, Baehrecke EH. Genome-wide analyses of steroid- and radiationtriggered programmed cell death in Drosophila. Curr Biol. 2003;13:350-7.

69. Lee CY, Baehrecke EH. Steroid regulation of autophagic programmed cell death during development. Development. 2001; 128:1443-55.

70. Berry DL, Baehrecke EH. Growth arrest and autophagy are required for salivary gland cell degradation in Drosophila. Cell. 2007;131:1137-48.

71. Fullgrabe J, Lynch-Day MA, Heldring N, Li W, Struijk RB, $\mathrm{Ma} \mathrm{Q}$, et al. The histone H4 lysine 16 acetyltransferase hMOF regulates the outcome of autophagy. Nature. 2013; 500:468-71.

72. Anding AL, Wang C, Chang TK, Sliter DA, Powers CM, Hofmann $\mathrm{K}$, et al. Vps13D encodes a ubiquitin-binding protein that is required for the regulation of mitochondrial size and clearance. Curr Biol. 2018;28:287-95 e6.

73. Denton D, Xu T, Dayan S, Nicolson S, Kumar S. Dpp regulates autophagy-dependent midgut removal and signals to block ecdysone production. Cell Death Differ. 2018. https://doi.org/10. 1038/s41418-018-0154-z.

74. Karch J, Schips TG, Maliken BD, Brody MJ, Sargent MA, Kanisicak $\mathrm{O}$, et al. Autophagic cell death is dependent on lysosomal membrane permeability through Bax and Bak. eLife. 2017;6:e30543.

75. Nezis IP, Shravage BV, Sagona AP, Lamark T, Bjorkoy G, Johansen T, et al. Autophagic degradation of dBruce controls DNA fragmentation in nurse cells during late Drosophila melanogaster oogenesis. J Cell Biol. 2010;190:523-31.

76. Hwang S, Maloney NS, Bruinsma MW, Goel G, Duan E, Zhang L, et al. Nondegradative role of Atg5-Atg12/ Atg16L1 autophagy protein complex in antiviral activity of interferon gamma. Cell Host Microbe. 2012;11:397-409.

77. Yousefi S, Perozzo R, Schmid I, Ziemiecki A, Schaffner T, Scapozza L, et al. Calpain-mediated cleavage of Atg5 switches autophagy to apoptosis. Nat Cell Biol. 2006;8:1124-32.

78. Zhu Y, Zhao L, Liu L, Gao P, Tian W, Wang X, et al. Beclin 1 cleavage by caspase-3 inactivates autophagy and promotes apoptosis. Protein Cell. 2010;1:468-77.

79. Maskey D, Yousefi S, Schmid I, Zlobec I, Perren A, Friis R, et al. ATG5 is induced by DNA-damaging agents and promotes mitotic catastrophe independent of autophagy. Nat Commun. 2013;4:2130.

80. Radoshevich L, Murrow L, Chen N, Fernandez E, Roy S, Fung $\mathrm{C}$, et al. ATG12 conjugation to ATG3 regulates mitochondrial homeostasis and cell death. Cell. 2010;142:590-600.

81. Grumati P, Dikic I. Ubiquitin signaling and autophagy. J Biol Chem. 2018;293:5404-13.

82. Gomez-Diaz C, Ikeda F. Roles of ubiquitin in autophagy and cell death. Semin Cell Dev Biol. 2018. https://doi.org/10.1016/j. semcdb.2018.09.004.

83. Khaminets A, Behl C, Dikic I. Ubiquitin-dependent and independent signals in selective autophagy. Trends Cell Biol. 2016; 26:6-16.

84. Thomas HE, Stunnenberg HG, Stewart AF. Heterodimerization of the Drosophila ecdysone receptor with retinoid $\mathrm{X}$ receptor and ultraspiracle. Nature. 1993;362:471-5.

85. Koelle MR, Talbot WS, Segraves WA, Bender MT, Cherbas P, Hogness DS. The Drosophila EcR gene encodes an ecdysone receptor, a new member of the steroid receptor superfamily. Cell. 1991;67:59-77.

86. Nicolson S, Denton D, Kumar S. Ecdysone-mediated programmed cell death in Drosophila. Int J Dev Biol. 2015; 59:23-32.

87. King-Jones K, Thummel CS. Nuclear receptors-a perspective from Drosophila. Nat Rev Genet. 2005;6:311-23.

88. Lee CY, Wendel DP, Reid P, Lam G, Thummel CS, Baehrecke EH. E93 directs steroid-triggered programmed cell death in Drosophila. Mol Cell. 2000;6:433-43.

89. Daish TJ, Cakouros D, Kumar S. Distinct promoter regions regulate spatial and temporal expression of the Drosophila caspase dronc. Cell Death Differ. 2003;10:1348-56.

90. Cakouros D, Daish TJ, Kumar S. Ecdysone receptor directly binds the promoter of the Drosophila caspase dronc// regulating its expression in specific tissues. J Cell Biol. 2004;165:631-40.

91. Cakouros D, Mills K, Denton D, Paterson A, Daish T, Kumar S. $\mathrm{dLKR} / \mathrm{SDH}$ regulates hormone-mediated histone arginine methylation and transcription of cell death genes. J Cell Biol. 2008;182:481-95.

92. Denton D, Aung-Htut MT, Lorensuhewa N, Nicolson S, Zhu W, Mills K, et al. UTX coordinates steroid hormone-mediated autophagy and cell death. Nat Commun. 2013;4:2916.

93. Tian L, Ma L, Guo E, Deng X, Ma S, Xia Q, et al. 20Hydroxyecdysone upregulates Atg genes to induce autophagy in the Bombyx fat body. Autophagy. 2013;9:1172-87.

94. Malta J, Heerman M, Weng JL, Fernandes KM, Martins GF, Ramalho-Ortigao M. Midgut morphological changes and autophagy during metamorphosis in sand flies. Cell Tissue Res. 2017;368:513-29. 
95. Neufeld TP. Autophagy and cell growth-the yin and yang of nutrient responses. J Cell Sci. 2012;125:2359-68.

96. Saxton RA, Sabatini DM. mTOR signaling in growth, metabolism, and disease. Cell. 2017;168:960-76.

97. Kamada Y, Funakoshi T, Shintani T, Nagano K, Ohsumi M, Ohsumi Y. Tor-mediated induction of autophagy via an Apg1 protein kinase complex. J Cell Biol. 2000;150:1507-13.

98. Neufeld TP. TOR-dependent control of autophagy: biting the hand that feeds. Curr Opin Cell Biol. 2010;22:157-68.

99. Denton D, Chang TK, Nicolson S, Shravage B, Simin R, Baehrecke EH, et al. Relationship between growth arrest and autophagy in midgut programmed cell death in Drosophila. Cell Death Differ. 2012;19:1299-307.

100. Rusten TE, Lindmo K, Juhasz G, Sass M, Seglen PO, Brech A, et al. Programmed autophagy in the Drosophila fat body is induced by ecdysone through regulation of the PI3K pathway. Dev Cell. 2004;7:179-92.

101. Xu T, Denton D, Kumar S. Hedgehog and Wingless signaling are not essential for autophagy dependent cell death. Biochem. Pharmacol.. 2018. https://doi.org/10.1016/j.bcp.2018.10.027.

102. Suzuki HI, Kiyono K, Miyazono K. Regulation of autophagy by transforming growth factor-beta (TGF-beta) signaling. Autophagy. 2010;6:645-7.

103. Kiyono K, Suzuki HI, Matsuyama H, Morishita Y, Komuro A, Kano MR, et al. Autophagy is activated by TGF-beta and potentiates TGF-beta-mediated growth inhibition in human hepatocellular carcinoma cells. Cancer Res. 2009;69:8844-52.

104. Xu Y, Yang S, Huang J, Ruan S, Zheng Z, Lin J. Tgf-beta1 induces autophagy and promotes apoptosis in renal tubular epithelial cells. Int J Mol Med. 2012;29:781-90.

105. Dutta S, Baehrecke EH. Warts is required for PI3K-regulated growth arrest, autophagy, and autophagic cell death in Drosophila. Curr Biol. 2008;18:1466-75.

106. McPhee CK, Logan MA, Freeman MR, Baehrecke EH. Activation of autophagy during cell death requires the engulfment receptor Draper. Nature. 2010;465:1093-6.
107. Lin L, Rodrigues F, Kary C, Contet A, Logan M, Baxter RHG, et al. Complement-related regulates autophagy in neighboring cells. Cell. 2017;170:158-71.e8.

108. Batlevi Y, Martin DN, Pandey UB, Simon CR, Powers CM, Taylor JP, et al. Dynein light chain 1 is required for autophagy, protein clearance, and cell death in Drosophila. Proc Natl Acad Sci USA. 2010;107:742-7.

109. Tracy K, Velentzas PD, Baehrecke EH. Ral GTPase and the exocyst regulate autophagy in a tissue-specific manner. EMBO Rep. 2016;17:110-21.

110. Nelson C, Ambros V, Baehrecke EH. miR-14 regulates autophagy during developmental cell death by targeting ip3-kinase 2 . Mol Cell. 2014;56:376-88.

111. Bodemann BO, Orvedahl A, Cheng T, Ram RR, Ou YH, Formstecher E, et al. RalB and the exocyst mediate the cellular starvation response by direct activation of autophagosome assembly. Cell. 2011;144:253-67.

112. Velentzas PD, Zhang L, Das G, Chang TK, Nelson C, Kobertz $\mathrm{WR}$, et al. The proton-coupled monocarboxylate transporter hermes is necessary for autophagy during cell death. Dev Cell. 2018;47:281-93 e4.

113. Santhanam A, Peng WH, Yu YT, Sang TK, Chen GC, Meng TC. Ecdysone-induced receptor tyrosine phosphatase PTP52F regulates Drosophila midgut histolysis by enhancement of autophagy and apoptosis. Mol Cell Biol. 2014;34:1594-606.

114. Meyer $\mathrm{H}$, Bug M, Bremer S. Emerging functions of the VCP/p97 AAA-ATPase in the ubiquitin system. Nat Cell Biol. 2012; 14:117-23.

115. Papadopoulos C, Kirchner P, Bug M, Grum D, Koerver L, Schulze N, et al. VCP/p97 cooperates with YOD1, UBXD1 and PLAA to drive clearance of ruptured lysosomes by autophagy. EMBO J. 2017;36:135-50.

116. Lindqvist LM, Frank D, McArthur K, Dite TA, Lazarou M, Oakhill JS, et al. Autophagy induced during apoptosis degrades mitochondria and inhibits type I interferon secretion. Cell Death Differ. 2018;25:782-94. 